\title{
Conditioned Medium from Adipose Tissue-Derived Mesenchymal Stem Cells Induces CD4+FOXP3+ Cells and Increases IL-10 Secretion
}

\author{
Ekaterina Ivanova-Todorova, ${ }^{1}$ Ivan Bochev, ${ }^{2}$ Rumen Dimitrov, ${ }^{3}$ \\ Kalina Belemezova, ${ }^{3}$ Milena Mourdjeva, ${ }^{3}$ Stanimir Kyurkchiev, ${ }^{2}$ \\ Plamen Kinov, ${ }^{4}$ Iskra Altankova, ${ }^{1}$ and Dobroslav Kyurkchiev ${ }^{1}$ \\ ${ }^{1}$ Laboratory of Clinical Immunology, University Hospital "St. Ivan Rilski," Medical University of Sofia, 15 Acad. Ivan Geshov Street, \\ 1431 Sofia, Bulgaria \\ ${ }^{2}$ Institute of Reproductive Health, Ob/Gyn Hospital “Dr. Shterev," 25-31 Hristo Blagoev Street, 1330 Sofia, Bulgaria \\ ${ }^{3}$ Laboratory of Molecular Immunology, Institute of Biology and Immunology of Reproduction, Bulgarian Academy of Sciences, \\ 73 Tsarigradsko Shosse Street, 1113 Sofia, Bulgaria \\ ${ }^{4}$ Department of Orthopedics and Traumatology, University Hospital "Tsaritsa Yoanna," Medical University of Sofia, \\ 8 Byalo More Street, 1527 Sofia, Bulgaria
}

Correspondence should be addressed to Dobroslav Kyurkchiev, dsk666@gmail.com

Received 12 June 2012; Accepted 5 October 2012

Academic Editor: Ken-ichi Isobe

Copyright () 2012 Ekaterina Ivanova-Todorova et al. This is an open access article distributed under the Creative Commons Attribution License, which permits unrestricted use, distribution, and reproduction in any medium, provided the original work is properly cited.

\begin{abstract}
Mesenchymal stem cells (MSCs) are a new and promising tool for therapy of autoimmune disorders. In recent years their possibility to take part in the modulation of the immune response is discussed. The exact mechanisms for immunoregulation realized by MSCs are not clear yet, but interactions with other immunoregulatory cells may be involved in this process. The investigation of the influence of MSCs on the expression of FoxP3 and cytokine secretion by T helper cells was the aim of this study. T helper cells were isolated from PBMCs by magnetic separation and MSCs were isolated from human adipose tissue, and CD4+ T cells were cultured with conditional medium of MSCs. The methods which were used include flow cytometry, ELISA, and Human Proteome profiler kits. The results demonstrated that secretory factors in MSCs conditional medium lead to increased expression of FoxP3 and increased secretion of IL-10 by T helpers. The obtained results give us opportunity to discuss the interaction between two kinds of immunoregulatory cells: MSCs and FoxP3+ T helpers. We suppose that this interaction leads to increased number of immunosuppressive helpers which secrete IL-10. MSCs provide some of their immunosuppressive functions acting on T regulatory cells, and we believe that IL- 6 secreted by MSCs is involved in this process.
\end{abstract}

\section{Introduction}

The process of immunomodulation is of utmost importance for sustaining the immune homeostasis in the organism, and detailed knowledge about the precise mechanisms of this process can bring about a better and more efficient therapy of the autoimmune diseases. Regulation of the immune response is accomplished by a number of cell subtypes and secreted factors as a lot of attention in the last years is focused on the role of the mesenchymal stem cells (MSCs) [1-3]. Numerous papers have reported data proving the important immunoregulatory functions of the human MSCs. It has been established that MSCs inhibit the proliferation of T and $\mathrm{B}$ cells, the production of $\mathrm{H}_{2} \mathrm{O}_{2}$ from neutrophils, the secretion of immunoglobulins, $\mathrm{T}$ and NK cytotoxicity as well as the differentiation and maturation of monocytes into dendritic cells. Also, the immunosuppression caused by the MSCs affects the secretion of cytokines which is biased to Th2 dominance with secretion of IL-10 and IL-4 while the secretion of IFN $\gamma$ and TNF $\alpha$ is suppressed [3-7]. The precise mechanisms of the MSCs immunosuppressive effect have not been clarified yet. Specific roles of secreted 
factors such as IDO, IL-6, TGF $\beta$, LIF, INOs, PGE2, HLA$\mathrm{G}[3,5-8]$ have been assigned, and the role of direct cellto-cell contact between the MSCs and the targeted cells is discussed $[9,10]$. However, besides data about the direct inhibitory effects of MSCs, there are data published on some indirect inhibitory effects which are mediated through influencing other immunoregulatory cells. Previous studies in our laboratory have demonstrated that MSCs inhibit the differentiation of monocytes to dendritic cells and downregulate the expression of molecules related with the antigen presentation and the expression of CCL-3 and CCL- 4 . These experiments showed that the effect of MSCs isolated from adipose tissue (AT-MSCs) is stronger in comparison to MSCs isolated from human bone marrow (BM-MSCs) [11]. Some indirect immunosuppressive effects of MSCs seem to be due to their influence on the classical immunoregulatory CD4+FoxP3+ cells generally referred to as Tregs. The last decade witnessed some significant development of the idea for the $\mathrm{T}$ regulatory cells. Along with the Th3 cells secreting TGF $\beta$ and $\operatorname{Tr} 1$ secreting IL-10 $[12,13]$ a role was established for $\mathrm{CD} 4+\mathrm{CD} 25+\mathrm{T}$ lymphocytes which exert immune suppression via CTLA-4, TGF $\beta$, and/or IL-10 [1417]. Later on an intracellular transcription factor FoxP3 was identified as a more precise marker for the $\mathrm{T}$ regulatory subpopulations [18-20]. It has been established that about $2 \%$ of CD4+ cells express FoxP3 [18], and it is related to the differentiation of Tregs and exerts direct functional immune suppression [13, 17, 21]. Recently, the role of CD25 molecule as a marker for suppressive subpopulations has raised some doubts. It is clear that the lack of surface expression of CD127 more accurately correlates with FoxP3, and this can be found even in CD25 negative cells $[18,22,23]$. Even more in patients with systemic lupus erythematosus (SLE) both CD4+CD25+FoxP3+ and CD4+CD25-FoxP3+ cells with expressed immunosuppressive properties have been identified $[24,25]$. That is the reason Tregs are more often designated as $\mathrm{CD} 4+\mathrm{FoxP} 3+$. The molecular mechanisms of immunosuppression exerted by $\mathrm{CD} 4+\mathrm{FoxP} 3+$ cells have not been identified but it is quite obvious that these mechanisms include the secretion of TGF $\beta$ and/or IL-10 [12, 17-19].

Some published data seem to show that under the influence of MSCs the number of the CD4+FoxP3+ cells is increased, and these cells produce the immunosuppressive cytokine IL-10, and thus MSCs exert indirect immunosuppressive effect $[2,7,9,10,26]$.

The aim of the present study is to analyze the effect of conditioned medium of AT-MSCs on the expression of FoxP3 molecule and the secretion of cytokines by a homogeneously purified subpopulation of CD4+ T lymphocytes. Additionally the factors secreted from the AT-MSCs are analyzed with the view of their putative effect on the changes in the $\mathrm{CD} 4+\mathrm{T}$ lymphocyte populations.

\section{Materials and Methods}

2.1. Materials. Samples of peripheral blood were collected by venepuncture from 12 healthy volunteers, and 12 samples of human adipose tissues were collected during hip surgery.
In both cases the samples were taken after a signed informed consent from the donors according to the regulations in this country.

2.2. Mesenchymal Stem Cells. Mesenchymal stem cells from adipose tissue were isolated, cultured, and phenotyped following strictly the generally accepted laboratory protocols and most of all the protocol developed in our laboratory [4].

2.3. Conditioned Medium. After forming a monolayer the AT-MSCs were detached after treatment with Trypsin EDTA $(1: 250)$ (PAA, Austria) and seeded in 6-well plate (passage 1) at concentration 3-5 $\times 10^{4}$ cell/well in DMEM medium supplement with $10 \%$ foetal bovine serum and antibiotics (PAA, Austria). The cells were cultured until reaching $80 \%$ confluency as the culture medium was changed every 48 hours. After the last change of the medium, the $80 \%$ confluent cells were further cultured for 48 hours, and the medium designated as "conditioned AT-MSC medium" was collected. Thus the conditioned AT-MSC medium contained DMEM, FBS, and any factors secreted by the nonactivated AT-MSCs. This procedure was strictly followed in 12 independent experiments performed with $\mathrm{T}$ cell isolated and purified from 12 individual donors and 12 different samples of conditioned medium obtained as described above.

2.4. Isolation of T Helper Lymphocytes. PBMCs (peripheral blood monocytic cells) were isolated from peripheral blood by density gradient centrifugation (Ficoll-Paque PLUS, GE Healthcare). The isolated cells were used for purification of CD4+ T lymphocytes using magnetic separation with MACS kits (Miltenyi Biotec, Bergisch-Gladbach, Germany). Initially, CD14+ monocytes were discarded from PBMCs by positive selection with anti-CD14-microbeads and the flow through fraction was collected. CD4+ cells were enriched from the resulting CD14- cell fraction using anti-CD4microbeads and the enriched CD4+ cell fraction was further characterized by flow cytometry mostly with regard to the expression of CD3/CD4 markers.

2.5. Cell Cultures. The isolated T helpers (Th) were cultured for 48 hours in conditioned medium from AT-MSCs at the first passage cultured for 48 hours. Control T helper cells were cultured in DMEM low glucose medium supplemented with $10 \%$ fetal bovine serum (PAA, Austria). Th cells were seeded at concentration $5 \times 10^{6}$ cells/well into 24 -well plates (PAA, Austria).

2.6. Flow Cytometry. Homogeneity of the purified $\mathrm{T}$ helper populations was proved using monoclonal antibodies antihuman CD3 FITC and anti-human CD4 PE. Anti-human CD4 FITC, anti-human HLA-DR PE, and anti-human CD69 PE antibodies were used to assess the surface expression of the activation markers by CD4+ T lymphocytes cultured in AT-MSCs conditioned medium as well as the control T helper cultures. Identification of $\mathrm{T}$ helper cells expressing surface CD25 and intracellular FoxP3 was performed using CD4 PerCP-Cy 5.5, anti-human CD25 FITC, anti-human 
FoxP3 PE and FoxP3 Buffer set following the standard procedure recommended by the producer company. All the antibodies and the machines (FACSCalibur) used were purchased from Becton Dickinson, CA, USA.

2.7. Enzyme Linked Immunosorbent Assay (ELISA). Supernatants from Th lymphocytes cultured with AT-MSCs conditioned medium or from control Th cell cultures as well as samples of AT-MSCs conditioned medium were tested for the presence of IL-2, IFN $\gamma$, IL-10, and TGFb by Human Instant ELISA (Bender MedSystems, Austria) following strictly the manufacturer's instructions.

2.8. Proteome Profiler Kits. Analysis of the cytokines in the AT-MSCs culture supernatants was performed using the Human Cytokine array panel A array kit (R\&D Systems, MN, USA) which can detect 36 different cytokines, chemokines, and growth factors (C5a, CD40L, G-CSF, GMCSF, GRO $\alpha$, I-309, sICAM-1, IFN $\gamma$, IL- $1 \alpha$, IL- $1 \beta$, IL-1ra, IL-2, IL-4, IL-5, IL-6, IL-8, IL-10, IL-12 p70, IL-13, IL-16, IL-17, IL-17E, IL-23, IL-27, IL-32 $\alpha$, IP-10, I-TAC, MCP-1, MIF, MIP- $1 \alpha$, MIP- $1 \beta$, Serpin E1, RANTES, SDF-1, TNF $\alpha$, TREM-1).

With the aim to assess any signs of apoptosis of the $\mathrm{CD} 4+\mathrm{T}$ cells cultured in AT-MSCs conditioned medium or CD4+ control cells the Proteome profiler human apoptosis kit (R\&D Systems, MN, USA) was used as this test is capable to establish the intracellular expression of Bad, Bax, Bcl-2, Bcl-x, Pro-Caspase-3, Cleaved Caspase-3, Catalase, clap-1, clap-2, Claspin, Clusterin, Cytochrome C, TRAIL R1/DR4, TRAIR R1/DR5, FADD, Fas/TNFSF6, HIF$1 \alpha$, HO-1/HMOX1/HPS32, HO-2/HMOX2, HSP27, HSP60, HSP70, HTRA2/Omi, Livin, PON2, p21/CIP1/CDNK1A, p27Kip1, Phospho-p53(S15), Phospho-p53(S46), Phosphop53(S392), Phospho-RAD17(S635), SMAC/Diablo, Survivin, TNF RI/TNFRSF1A, XIAP.

2.9. Software. The programs CellQuest and WinMDI 2.9 were used for analysis of the flow cytometry data. Image J program (NIH, Bethesda, MD, USA) was used for analysis of the results from the Human Cytokine array panel A array kit and Proteome profiler human apoptosis kit.

2.10. Statistics. Statistical analyses of cytokine concentrations were performed using paired Student's $t$-test. Data were presented as mean \pm standard deviation (SD). Statistical significance was established at $P<0.05$.

\section{Results}

3.1. Secreted Factors Present in AT-MSCs Conditioned Medium Cause an Increase of the Numbers of T Helper Cells Expressing FoxP3. The procedure followed in our experiments leads to isolation of a very homogeneous population of $\mathrm{CD} 3+\mathrm{CD} 4+$ T helper lymphocytes (Figures 1(a) and 1(b)). Cultivation of these cells in AT-MSCs conditioned medium did not cause any alterations in the expression of the activation markers CD69 and HLA-DR (data not shown). However, cultures of Th cells in conditioned medium caused an increase of the numbers of CD4+ T lymphocytes expressing FoxP3 (Figure 1(d)) as compared to the same parameter in control cells (Figure 1(c)). This effect was found for both $\mathrm{T}$ helpers positive for the surface expression of CD25 and for T helpers negative for CD25 and was recorded in all samples tested from individual donors (Table 1). So, it is obvious that some secreted factors present in AT-MSCs conditioned medium induce a specific upregulation of the transcription factor FoxP3 which defines the Treg subpopulation regardless of the expression of CD25.

\subsection{Secreted Factors Present in AT-MSCs Conditioned Medium} Caused an Upregulation of the IL-10 Secretion by T Helper Lymphocytes. The search of secreted cytokines revealed the presence of TGF $\beta$ in very low concentrations in both $\mathrm{CD} 4+\mathrm{T}$ cells cultured in AT-MSCs conditioned medium and control CD4+ $\mathrm{T}$ medium samples in all cases tested (Figure 2(a)). No statistically significant differences were found for the concentrations of IFN $\gamma$ or IL-2 in experimental or control samples in each experiment with donor lymphocytes (Figures 2(b) and 2(c)). In contrast to these findings statistically significant higher concentration of IL-10 was detected in supernatants from CD4+ T cells cultured in AT-MSCs conditioned medium in comparison to the control supernatants (Figure 2(d)). This increased secretion of IL-10 was recorded in all cases of Th cells cultured in AT-MSCs conditioned medium. It should be pointed out that the ATMSCs conditioned medium itself did not contain any IL-10, and this gives us ground to conclude that the increased concentration of this cytokine by T helpers cultured in AT-MSCs conditioned medium is due to its secretion by activated $\mathrm{Th}$ lymphocytes.

3.3. Secreted Factors in AT-MSCs Conditioned Medium Did Not Induce any Changes in the Pro- and Antiapoptotic Factors in T Helper Lymphocytes. One of the possible ways for MSCs to alter the $\mathrm{T}$ helper cells is the influence of the process of apoptosis, and in order to check this possibility the presence and changes of the intracellular proapoptotic or antiapoptotic factors were assayed in the same experimental design. However, no significant differences were found between Th cells cultured in AT-MSCs medium and the control cells (data not shown).

3.4. AT-MSCs Conditioned Medium Contains Some Chemokines and IL-6. Repeated testing of the AT-MSCs conditioned medium showed that it contained several chemokines with known effect on $\mathrm{T}$ helper cells and just a single cytokine with proven immunoregulatory effect. The chemokines were Gro $\alpha$, RANTES, Serpin E, IL-8, and SDF-1 and from the cytokines the IL- 6 was found to be with quite high concentration in the AT-MSCs conditioned medium. These chemokines and IL-6 were found in negligible concentrations in control culture medium which point to their secretion by AT-MSCs (Figure 3). 


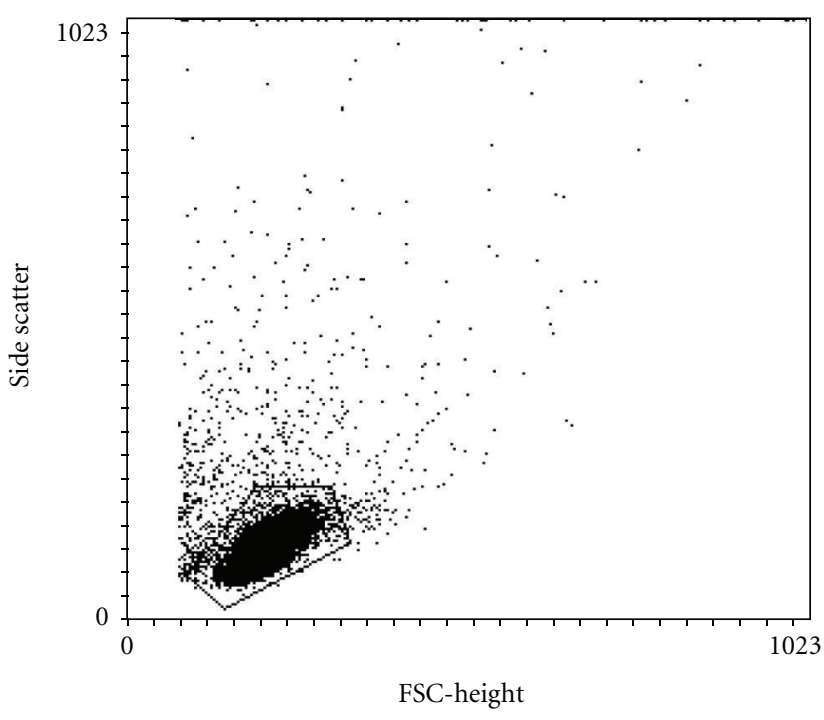

(a)

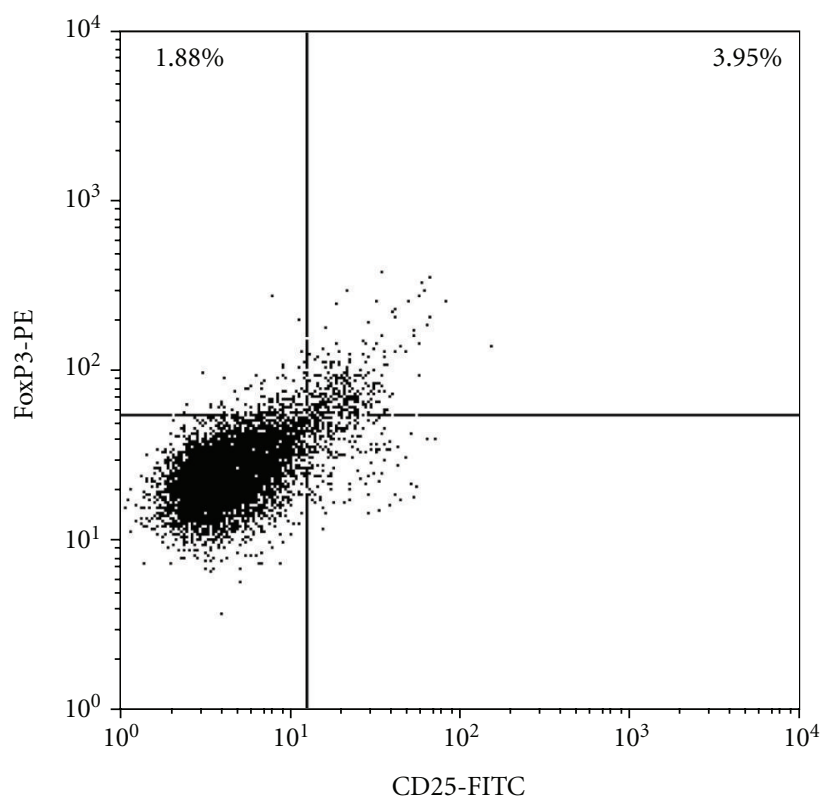

(c)

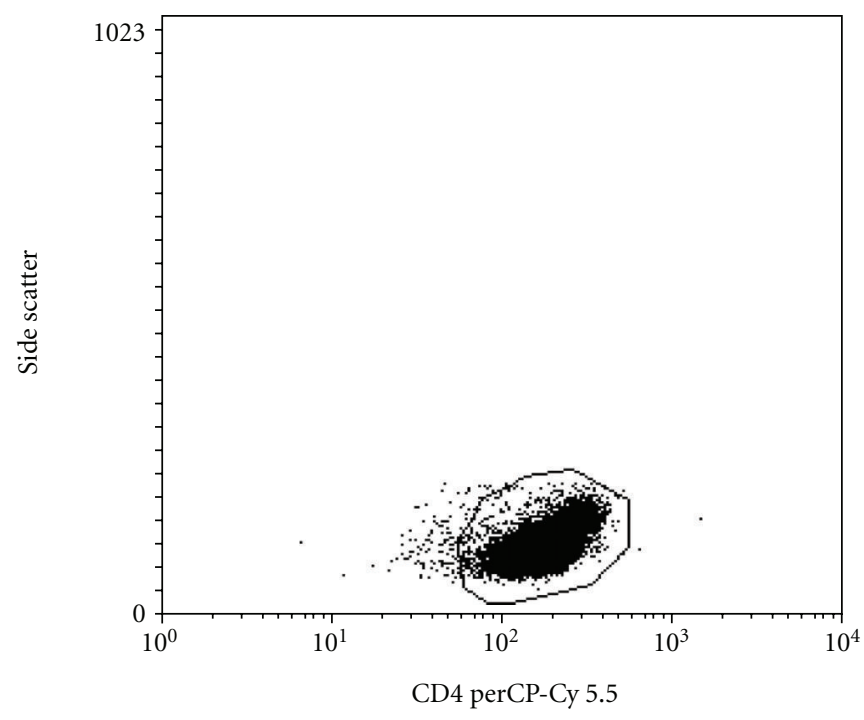

(b)

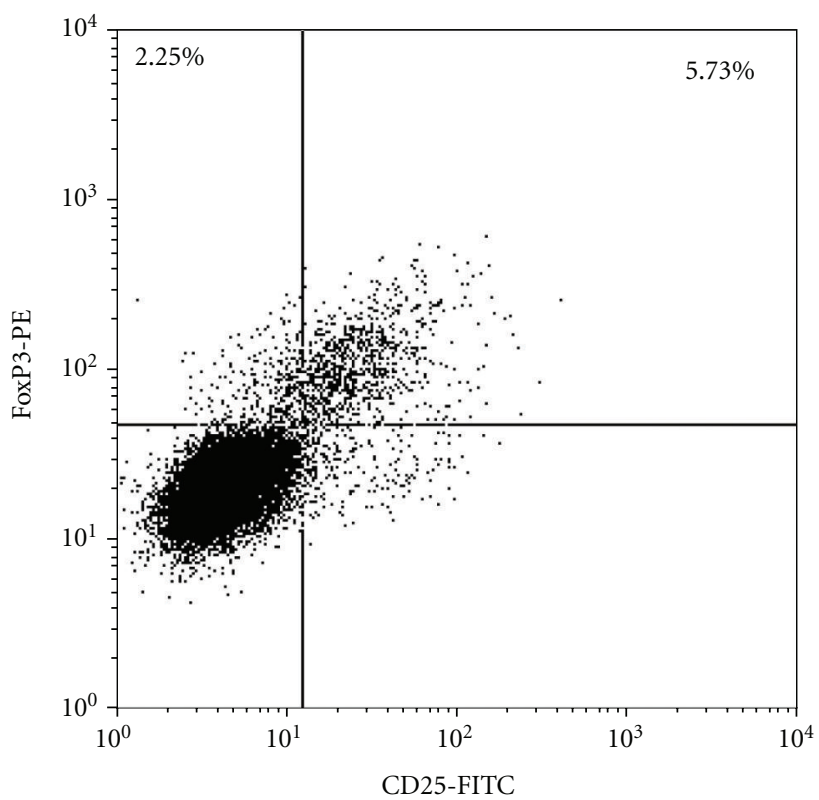

(d)

Figure 1: A representative dot plot showing the expression of CD25 and FoxP3 markers by T helpers. Panels (a) and (b) show the homogeneity of the purified CD4+ population; (c) shows the percentage of FoxP3+ cells in control culture; (d) shows an increase of the percentage of cells expressing FoxP3 after being cultured in AT-MSCs conditioned medium.

\section{Discussion}

The results obtained showed that when homogeneous population of CD4+ $\mathrm{T}$ lymphocytes was cultured in ATMSCs conditioned medium, the percentage of the $\mathrm{T}$ helpers expressing FoxP3 is increased as both CD25+ and CD25- cell subpopulations were affected. The role of the CD4+CD25+FoxP3+ cells as the major immunosuppressive factor has been known for a rather long time. It has been described that these cells can originate directly from the thymus and are designated as natural (nTregs) or can differentiate under the influence of cytokines in the periphery and are called induced (iTregs) $[13,19,27]$. The major cytokines responsible for inducing the differentiation of iTregs are most probably IL-2 and TGF $\beta$ [28]. We could not detect these cytokines in the AT-MSCs conditioned medium, but judging on the increased percentage of CD4+CD25+FoxP3+ it can be speculated that other cytokines may be involved in this process as discussed in the following.

Further on, our results revealed that under the influence of AT-MSCs conditioned medium, the percentages of the recently described subpopulation CD4+CD25-FoxP3+ increase as well. These cells were found to be increased in SLE patients and were considered to be progenitors of CD25+ 
TABLE 1: The effect of AT-MSCs conditioned medium on the percentage values of CD4+FoxP3+ T lymphocytes in cultures of T helper cells obtained from different donors. The data from all subjects tested revealed the stable tendency towards an increase of CD4+CD25+FoxP3+ and CD4+CD25-FoxP3+ cell numbers in the presence of AT-MSCs conditioned medium compared with the respective control cultures.

\begin{tabular}{lcccc}
\hline & \multicolumn{2}{c}{ CD4+CD25+FoxP3+ } & \multicolumn{2}{c}{ CD4+CD25-FoxP3+ } \\
& w/o AT-MSCs c.m. & With AT-MSCs c.m. & w/o AT-MSCs c.m. & With AT-MSCs c.m. \\
\hline Donor 1 & $3.45 \%$ & $4.42 \%$ & $2.12 \%$ & $4.12 \%$ \\
Donor 2 & $2.19 \%$ & $2.91 \%$ & $1.72 \%$ & $2.37 \%$ \\
Donor 3 & $5.04 \%$ & $5.52 \%$ & $1.74 \%$ & $2.26 \%$ \\
Donor 4 & $1.47 \%$ & $2.48 \%$ & $2.84 \%$ & $3.06 \%$ \\
Donor 5 & $3.95 \%$ & $5.73 \%$ & $1.88 \%$ & $2.25 \%$ \\
Donor 6 & $2.87 \%$ & $4.55 \%$ & $1.01 \%$ & $1.95 \%$ \\
Donor 7 & $5.80 \%$ & $7.65 \%$ & $2.84 \%$ & $4.32 \%$ \\
Donor 8 & $5.69 \%$ & $5.81 \%$ & $3.13 \%$ & $3.78 \%$ \\
Donor 9 & $1.30 \%$ & $3.33 \%$ & $1.41 \%$ & $2.32 \%$ \\
Donor 10 & $1.77 \%$ & $2.03 \%$ & $0.7 \%$ & $1.10 \%$ \\
Donor 11 & $1.90 \%$ & $5.39 \%$ & $0.7 \%$ & $0.92 \%$ \\
Donor 12 & $2.92 \%$ & $5.06 \%$ & $1.6 \%$ & $1.7 \%$ \\
\hline
\end{tabular}

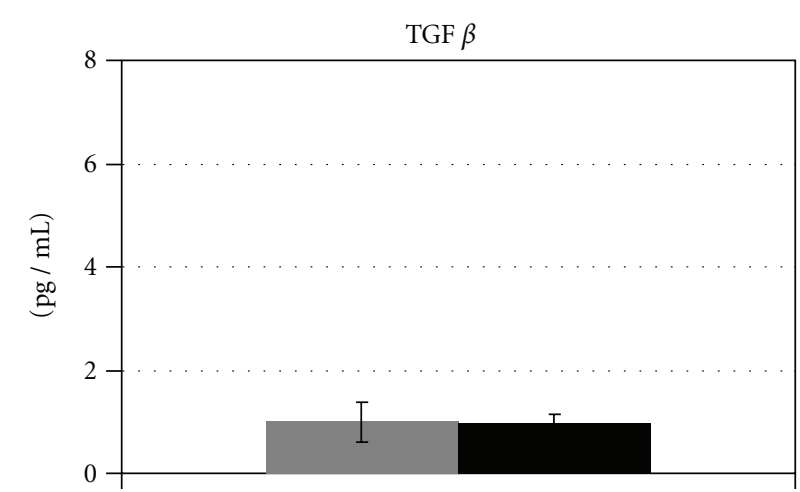

(a)

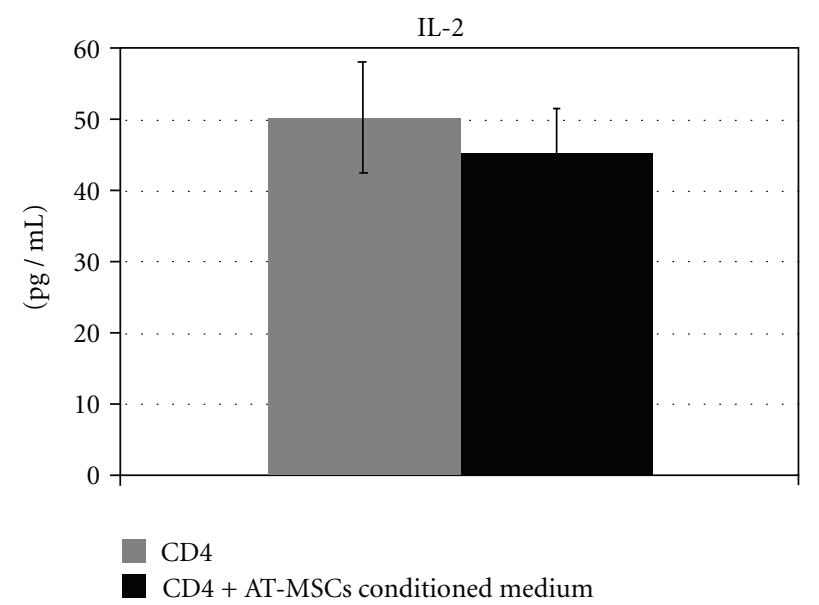

(c)

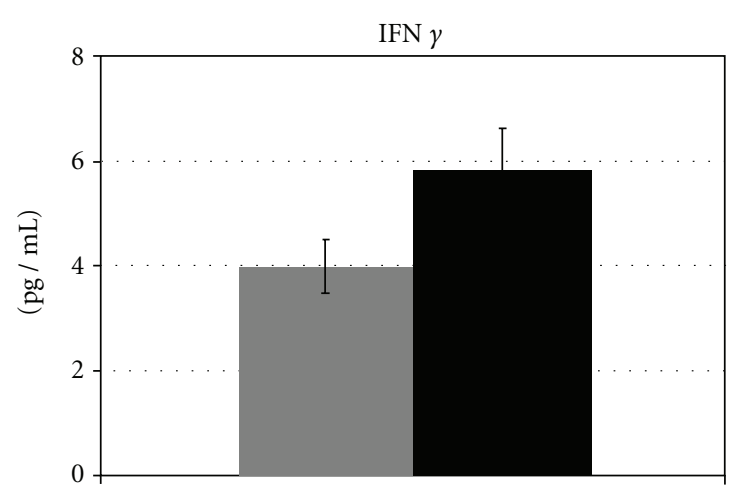

(b)

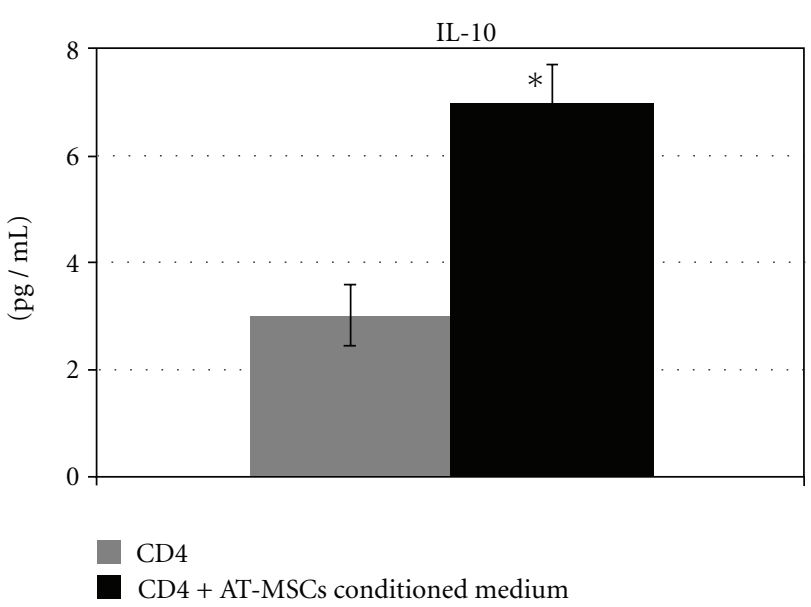

(d)

FIgURE 2: Cytokine secretion by CD4+ T lymphocytes cultured in AT-MSCs conditioned medium or control DMEM medium. Cultivation of CD4+ T cells in AT-MSCs conditioned medium did not induce changes in the secretion of TGF $\beta$ (a), IFN $\gamma$ (b), or IL-2 (c). In contrast the secretion of IL-10 was statistically significantly upregulated $\left({ }^{*} P<0.05\right)$ in the presence of AT-MSCs conditioned medium compared with the control (d). Mean values \pm SD of 12 independent experiments with cells from different donors are shown. 


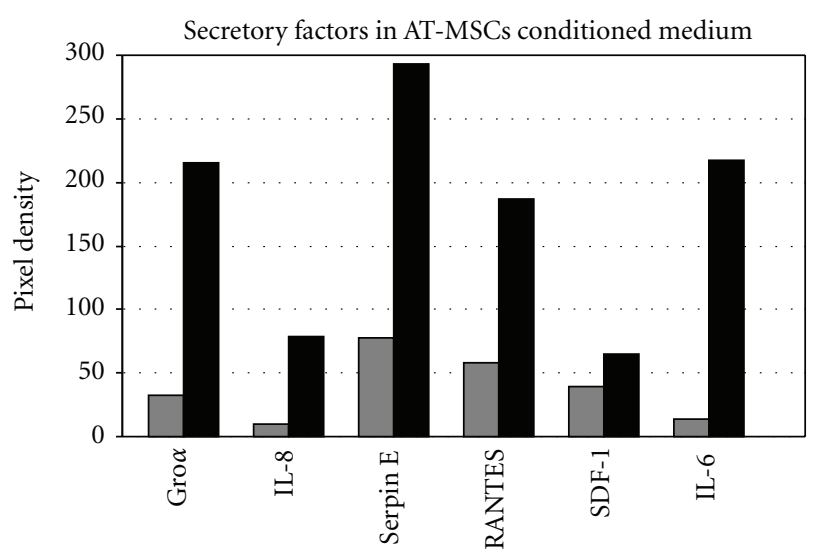

Figure 3: Cytokines and chemokines found to be present at significant levels in AT-MSCs conditioned medium (black columns) compared to control DMEM medium (grey columns). The figure represents only the cytokines and chemokines which are known to affect $\mathrm{T}$ helper activities.

cells [24]. Different opinions are discussed in the specialized literature as some authors expressed some doubts whether this subpopulation is immunoregulatory by itself [18], but the prevailing opinion is independently to the expression of CD25, the FoxP3 by itself exert functional suppression $[13,17,21,29]$. Based on this assumption the regulatory cells are more often designated as $\mathrm{CD} 4+\mathrm{FoxP} 3+[18,19]$. It is commonly accepted that the CD4+FoxP3+ cells express their immunosuppressive effects through the secretion of TGF $\beta$ and/or IL-10 [12, 15, 17, 18]. In our studies we did not record an increased secretion of TGF $\beta$ by CD4+ T lymphocytes cultured in AT-MSCs medium. It is quite possible that under experimental design used in our experiments this cytokine is being expressed as membrane-bound form at the surface of the CD4+ $\mathrm{T}$ lymphocytes as such a mechanism has been described for CD4+FoxP3+ cells and still is able to express its suppressive effect $[14,19]$. However, statistically significant increase was found for IL-10 secreted by CD4+ T lymphocytes cultured in AT-MSCs conditioned medium. The secretion of IL- 10 by CD4+FoxP3+ cells is considered to be one of the most important mechanisms via which this cell subpopulation exerts the immunosuppressive function $[30,31,31]$. IL-10 is an anti-inflammatory cytokine which inhibits the secretion of proinflammatory cytokines by the macrophages and dendritic cells [30]. It inhibits the activities of Th17 [31] and the differentiation of blood monocytes into dendritic cells $[11,30]$. One of the most significant immunoregulatory effects is the induction of tolerance in dendritic cells by inhibition of molecules which are crucial for antigenic presentation such as the B7 complex [11]. Moreover, IL-10 directly inhibits the CD28 expression on Th surface, and this molecule is the specific ligand for the B7 complex. Our previous results have shown that AT-MSCs upregulate the secretion of IL-10 by monocytic dendritic cells [11]. However, under the experimental conditions described it cannot be claimed that the CD4+FoxP3+ cells are the only source of the IL-10, but having in mind the

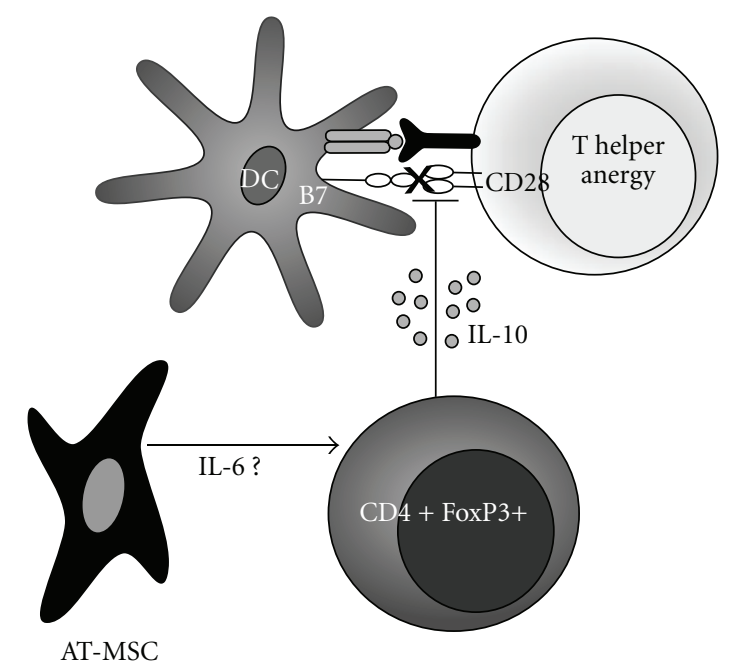

FIGURE 4: A possible indirect mechanism of the immunoregulatory effects of factors secreted by AT-MSCs.

increased number of these cells it is quite possible that there is a causative link between the increased numbers of $\mathrm{CD} 4+\mathrm{FoxP} 3+$ cells and the increased concentrations of IL10 in the culture medium. It can be further speculated that under the influence of the AT-MSCs conditioned medium the number of CD4+FoxP3+ cells secreting IL-10 increases, and this could lead to an inhibition of the costimulatory molecule expression on the surface of the dendritic cells and $\mathrm{T}$ helpers which is the most important condition for inducing anergy in the $\mathrm{T}$ helper cells. Therefore, together with the other mechanisms for immune suppression the AT-MSCs realize an indirect immune suppression by influencing other immunomodulatory cells such as dendritic cells and CD4+FoxP3+ lymphocytes (Figure 4). AT-MSCs induce secretion of IL-10 by CD4+FoxP3+ T cells. This cytokine inhibits the expression of key molecules of antigen presentation, and this process leads to anergy of the $\mathrm{T}$ lymphocytes. It can be speculated that IL- 6 secreted by AT-MSCs is one of the key elements responsible for this effect.

It is known that in parallel to its anti-inflammatory effect the IL-10 has proapoptotic effect on dendritic cells as shown in a previous paper [11]. The present studies did not find any changes with regard to the expression of the intracellular proor antiapoptotic factors in CD4+ T lymphocytes cultured in the presence of AT-MSCs conditioned medium. It has been reported that the MSCs in general realize their immunomodulatory functions either through direct contact between the cells [6] or through secretion of cytokines.

As mentioned above AT-MSCs conditioned medium was used in our experiments which means that the observed effects are due to the presence of secreted factors in the medium. Chemokines such as Gro $\alpha$, RANTES, Serpin E, IL-8, and SDF-1 which influence the chemotaxis of the T lymphocytes were shown to be present in the conditioned medium samples. So, it can be assumed that the process of immunosuppression realized by the AT-MSCs would be 
initiated with recruiting the T lymphocytes as the first step of the process.

Several types of secretory factors secreted by MSCs have been reported in the literature which can influence the formation of CD4+FoxP3+ cells, and these include IDO, TGF $\beta$, INOs, PGE2, HLA-G5, I-309, IL-10, IL-6 [6-9, 31].

In our experiments the presence of IL-6 solely was recorded in samples of AT-MSCs conditioned medium although we searched for TGF $\beta$, I-309, and IL-10, too. A probable explanation of this fact would be the assumption that MSCs secrete a number of cytokines after being stimulated by other cytokines [3]. Thus, after stimulation with IFN $\gamma$ the MSCs secrete I-309 which on its side induces the formation of Tregs [8] while the direct contact and IL10 secretion have a key role in induction of the secretion of HLA-G5 which would have the same effect [9]. Our results show that such an induction is not needed for the secretion of IL- 6 by AT-MSCs. The generally accepted views for IL- 6 hold that it is a proinflammatory cytokine which has a role in inhibition of the Tregs and stimulation of the Th17 immune response $[18,32]$. However other data would suppose that the function of IL- 6 should be reassessed because of its reported immunosuppressive activity. IL-6 causes increased secretion of IL-10 and can exert antiapoptotic activity [ 7,33$]$. It has been reported that MSCs secrete high levels of IL6 , and this secretion correlates directly with inhibition of the $\mathrm{T}$ cells, inhibition of the differentiation of the dendritic cells, and inhibition of the secretion of proinflammatory cytokines as well [34]. Similarly, the IL-6 increases the secretion of other immunosuppressive factors such as INOs and PGE2 by MSCs acting by an autocrine mechanism [35] and also directly causes an increase of the numbers of newly described CD8+FoxP3+ immunoregulatory cells $[29,36]$. The numerous activities of the IL-6 mentioned give ground to discuss its role as an anti-inflammatory cytokine. In agreement with the above cited data and our own results it can be speculated that under the influence of the IL- 6 secreted by nonstimulated AT-MSCs the number of IL-10 secreting CD4+FoxP3+ cells increases, and thus AT-MSCs realize an indirect immunosuppressive effect via the Tregs. Really it should not be claimed that the IL- 6 is the only one cytokine in this process since most of the factors modulating CD4+FoxP3+ cell functions have not been tested in our experiments. Most probably the cytokine induction of MSCs to secrete immunosuppressive factors as well as the direct cell-to-cell contacts with the subsequent secretion of HLAG5 would be of high importance. Previous results from our laboratory have shown the presence of HLA-G5 in the cytoplasm of AT-MSCs [37] as the effect of this molecule with regard to the Tregs cells has been described in several papers $[1,5-7]$.

\section{Conclusion}

In conclusion, the results obtained show that under the influence of factors secreted by AT-MSCs the numbers of CD4+FoxP3+ lymphocytes are increased and the secretion of IL-10 is upregulated in purified CD4+ T cell population.
It can be supposed that the presence of IL-6 is very important for this process. Similar effects of MSCs on the FoxP3+ cells and secretion of IL-10 have been described in other papers $[2,6-9]$ but bone-marrow-derived MSCs and umbilical cord-derived MSCs have been used, and they were cocultured with heterogeneous cell mixture of PBMCs. Original features in our experiments are the use of ATMSCs conditioned medium and a homogeneous population of CD4+ T lymphocytes. Under these conditions there is no direct cell-to-cell contact which might activate the AT-MSCs by other cell types, and the "native" or "passive" secretion of cytokines by AT-MSCs and their effects on the purified T helper cells were observed.

\section{References}

[1] P. Fiorina, M. Jurewicz, A. Augello et al., "Immunomodulatory function of bone marrow-derived mesenchymal stem cells in experimental autoimmune type 1 diabetes," Journal of Immunology, vol. 183, no. 2, pp. 993-1004, 2009.

[2] Y. Liu, R. Mu, S. Wang et al., "Therapeutic potential of human umbilical cord mesenchymal stem cells in the treatment of rheumatoid arthritis," Arthritis Research and Therapy, vol. 12, no. 6, p. R210, 2010.

[3] Y. Shi, G. Hu, J. Su et al., "Mesenchymal stem cells: a new strategy for immunosuppression and tissue repair," Cell Research, vol. 20, no. 5, pp. 510-518, 2010.

[4] I. Bochev, G. Elmadjian, D. Kyurkchiev et al., "Mesenchymal stem cells from human bone marrow or adipose tissue differently modulate mitogen-stimulated B-cell immunoglobulin production in vitro," Cell Biology International, vol. 32, no. 4, pp. 384-393, 2008.

[5] F. Djouad, C. Bouffi, S. Ghannam, D. Noël, and C. Jorgensen, "Mesenchymal stem cells: innovative therapeutic tools for rheumatic diseases.," Nature Reviews, vol. 5, no. 7, pp. 392399, 2009.

[6] E. Bassi, C. Aita, and N. Camara, "Immune regulatory properties of multipotent mesenchymal stromal cells: where do we stand," World Journal of Stem Cells, vol. 3, no. 1, pp. 1-8, 2011.

[7] E. Ben-Ami, S. Berrih-Aknin, and A. Miller, "Mesenchymal stem cells as an immunomodulatory therapeutic strategy for autoimmune diseases," Autoimmunity Reviews, vol. 10, no. 7, pp. 410-415, 2011.

[8] R. E. Newman, D. Yoo, M. A. LeRoux, and A. DanilkovitchMiagkova, "Treatment of inflammatory diseases with mesenchymal stem cells," Inflammation and Allergy, vol. 8, no. 2, pp. 110-123, 2009.

[9] Z. Selmani, A. Naji, I. Zidi et al., "Human leukocyte antigenG5 secretion by human mesenchymal stem cells is required to suppress $\mathrm{T}$ lymphocyte and natural killer function and to induce CD4+ CD25highFOXP3+ regulatory T cells," Stem Cells, vol. 26, no. 1, pp. 212-222, 2008.

[10] Z. Ye, Y. Wang, H. Y. Xie, and S. S. Zheng, "Immunosuppressive effects of rat mesenchymal stem cells: involvement of CD4+CD25+ regulatory T cells," Hepatobiliary and Pancreatic Diseases International, vol. 7, no. 6, pp. 608-614, 2008.

[11] E. Ivanova-Todorova, I. Bochev, M. Mourdjeva et al., "Adipose tissue-derived mesenchymal stem cells are more potent suppressors of dendritic cells differentiation compared to bone marrow-derived mesenchymal stem cells," Immunology Letters, vol. 126, no. 1-2, pp. 37-42, 2009. 
[12] W. Zou, "Regulatory T cells, tumour immunity and immunotherapy," Nature Reviews Immunology, vol. 6, no. 4, pp. 295$307,2006$.

[13] L. R. Guerin, J. R. Prins, and S. A. Robertson, "Regulatory Tcells and immune tolerance in pregnancy: a new target for infertility treatment?" Human Reproduction Update, vol. 15, no. 5, pp. 517-535, 2009.

[14] D. Dieckmann, C. H. Bruett, H. Ploettner, M. B. Lutz, and G. Schuler, "Human CD4+CD25+ regulatory, contactdependent $\mathrm{T}$ cells induce interleukin 1-producing, contactindependent type 1-like regulatory T cells," Journal of Experimental Medicine, vol. 196, no. 2, pp. 247-253, 2002.

[15] C. Baecher-Allan, V. Viglietta, and D. A. Hafler, "Human CD4+CD25+ regulatory T cells," Seminars in Immunology, vol. 16, no. 2, pp. 89-97, 2004.

[16] T. L. Holm, J. Nielsen, and M. H. Claesson, "CD4+CD25+ regulatory T cells: I. Phenotype and physiology," APMIS, vol. 112, no. 10, pp. 629-641, 2004.

[17] H. Jiang and L. Chess, "An integrated view of suppressor T cell subsets in immunoregulation," Journal of Clinical Investigation, vol. 114, no. 9, pp. 1198-1208, 2004.

[18] D. A. Horwitz, "Regulatory T cells in systemic lupus erythematosus: past, present and future," Arthritis Research and Therapy, vol. 10, no. 6, p. 227, 2008.

[19] R. E. Cone and S. Bhowmick, "Cytokines and sympathy: the control of regulatory T cells," International Journal of Interferon, Cytokine and Mediator Research, vol. 2, no. 1, pp. 41-47, 2010.

[20] J. A. Bluestone, "Mechanisms of tolerance," Immunological Reviews, vol. 241, no. 1, pp. 5-19, 2011.

[21] A. Y. Rudensky, "Regulatory T cells and Foxp3," Immunological Reviews, vol. 241, no. 1, pp. 260-268, 2011.

[22] W. Liu, A. L. Putnam, Z. Xu-yu et al., "CD127 expression inversely correlates with FoxP3 and suppressive function of human CD4+ T reg cells," Journal of Experimental Medicine, vol. 203, no. 7, pp. 1701-1711, 2006.

[23] L. S. Shen, J. Wang, D. F. Shen et al., "CD4+CD25+CD127 low/- regulatory $\mathrm{T}$ cells express Foxp3 and suppress effector $\mathrm{T}$ cell proliferation and contribute to gastric cancers progression," Clinical Immunology, vol. 131, no. 1, pp. 109-118, 2009.

[24] B. Yan and Y. Liu, "The nature of increased circulating CD4+CD25 -Foxp3+ T cells in patients with systemic lupus erythematosus: a novel hypothesis," Open Rheumatology Journal, vol. 3, pp. 22-24, 2009.

[25] D. A. Horwitz, "Identity of mysterious CD4+CD25-Foxp3+ cells in systemic lupus erythematosus," Arthritis Research and Therapy, vol. 12, no. 1, p. 101, 2010.

[26] E. Gonzalez-Rey, P. Anderson, M. A. González, L. Rico, D. Büscher, and M. Delgado, "Human adult stem cells derived from adipose tissue protect against experimental colitis and sepsis," Gut, vol. 58, no. 7, pp. 929-939, 2009.

[27] S. F. Ziegler and J. H. Buckner, "FOXP3 and the regulation of Treg/Th17 differentiation," Microbes and Infection, vol. 11, no. 5, pp. 594-598, 2009.

[28] M. A. de Lafaille and J. J. Lafaille, "Natural and adaptive Foxp3+ regulatory T cells: more of the same or a division of labor?" Immunity, vol. 30, no. 5, pp. 626-635, 2009.

[29] T. Nakagawa, M. Tsuruoka, H. Ogura et al., "IL-6 positively regulates Foxp3+CD8+ T cells in vivo," International Immunology, vol. 22, no. 2, Article ID dxp119, pp. 129-139, 2009.

[30] A. O'Garra and P. Vieira, "TH1 cells control themselves by producing interleukin-10," Nature Reviews Immunology, vol. 7, no. 6, pp. 425-428, 2007.
[31] Y. J. Heo, Y. B. Joo, H. J. Oh et al., "IL-10 suppresses Th17 cells and promotes regulatory $\mathrm{T}$ cells in the $\mathrm{CD} 4+\mathrm{T}$ cell population of rheumatoid arthritis patients," Immunology Letters, vol. 127, no. 2, pp. 150-156, 2010.

[32] A. Kimura and T. Kishimoto, "IL-6: regulator of Treg/Th17 balance," European Journal of Immunology, vol. 40, no. 7, pp. 1830-1835, 2010.

[33] A. Steensberg, C. P. Fischer, C. Keller, K. Møller, and B. K. Pedersen, "IL-6 enhances plasma IL-1ra, IL-10, and cortisol in humans," American Journal of Physiology-Endocrinology and Metabolism, vol. 285, no. 2, pp. E433-E437, 2003.

[34] F. Djouad, L. M. Charbonnier, C. Bouffi et al., "Mesenchymal stem cells inhibit the differentiation of dendritic cells through an interleukin-6-dependent mechanism," Stem Cells, vol. 25, no. 8, pp. 2025-2032, 2007.

[35] C. Bouffi, C. Bony, G. Courties, C. Jorgensen, and D. Noël, "IL-6-dependent PGE2 secretion by mesenchymal stem cells inhibits local inflammation in experimental arthritis," PLoS ONE, vol. 5, no. 12, Article ID e14247, 2010.

[36] C. T. Mayer, S. Floess, A. M. Baru, K. Lahl, J. Huehn, and T. Sparwasser, "CD8+Foxp3+ T cells share developmental and phenotypic features with classical CD4+Foxp3+ regulatory $\mathrm{T}$ cells but lack potent suppressive activity," European Journal of Immunology, vol. 41, no. 3, pp. 716-725, 2011.

[37] E. Ivanova-todorova, M. Mourdjeva, D. Kyurkchiev et al., "HLA-G expression Is up-regulated by progesterone in mesenchymal stem cells," American Journal of Reproductive Immunology, vol. 62, no. 1, pp. 24-33, 2009. 

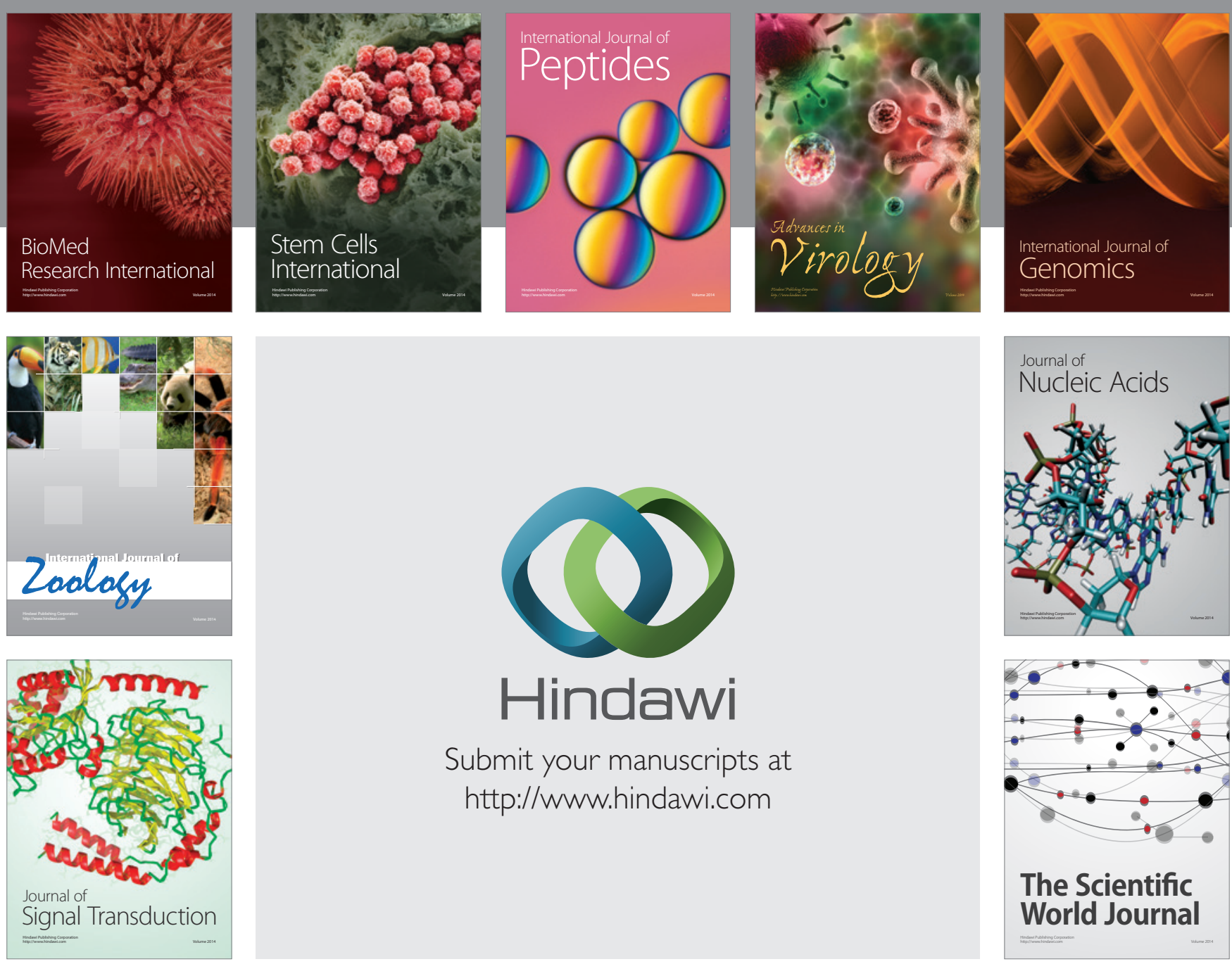

Submit your manuscripts at

http://www.hindawi.com
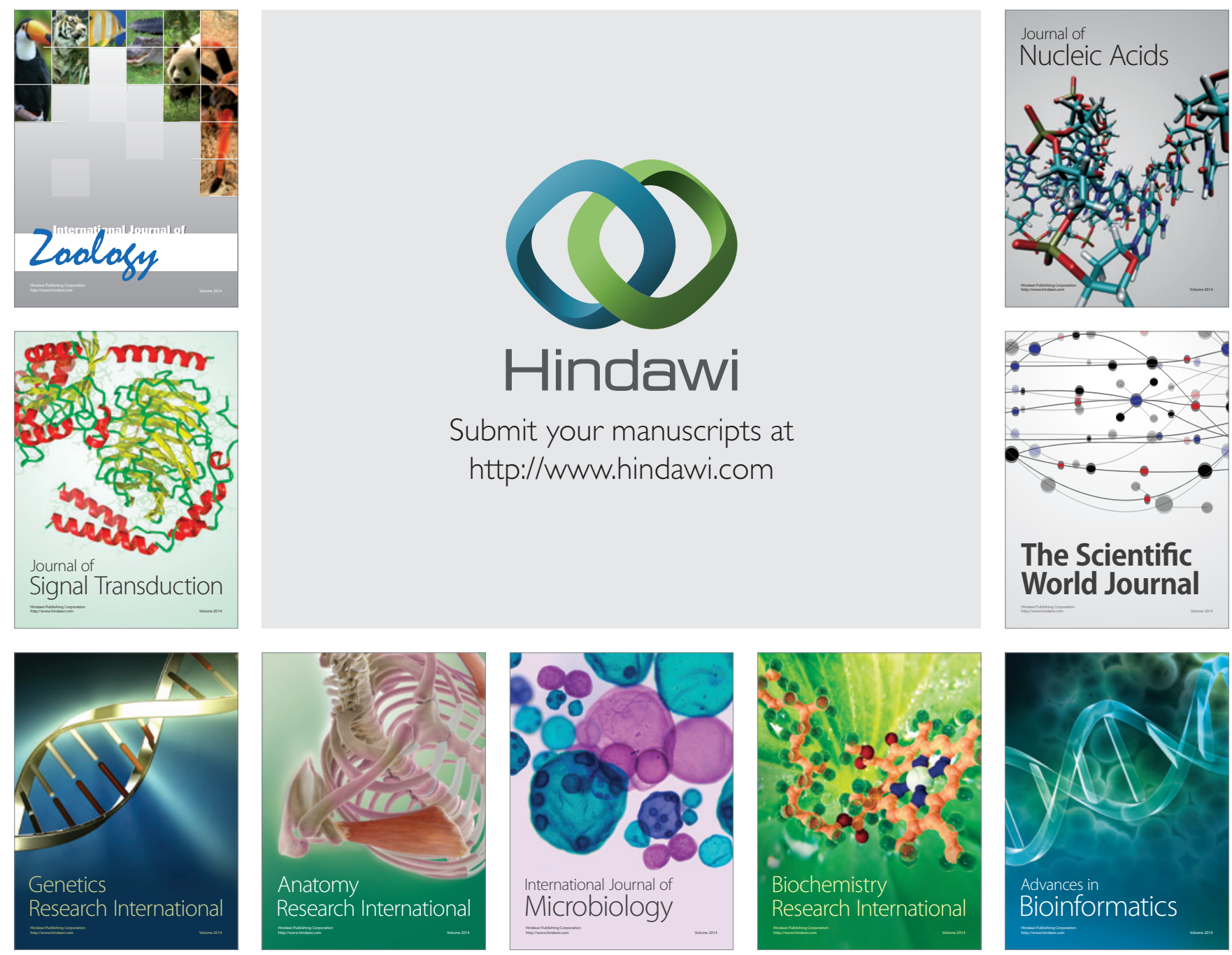

The Scientific World Journal
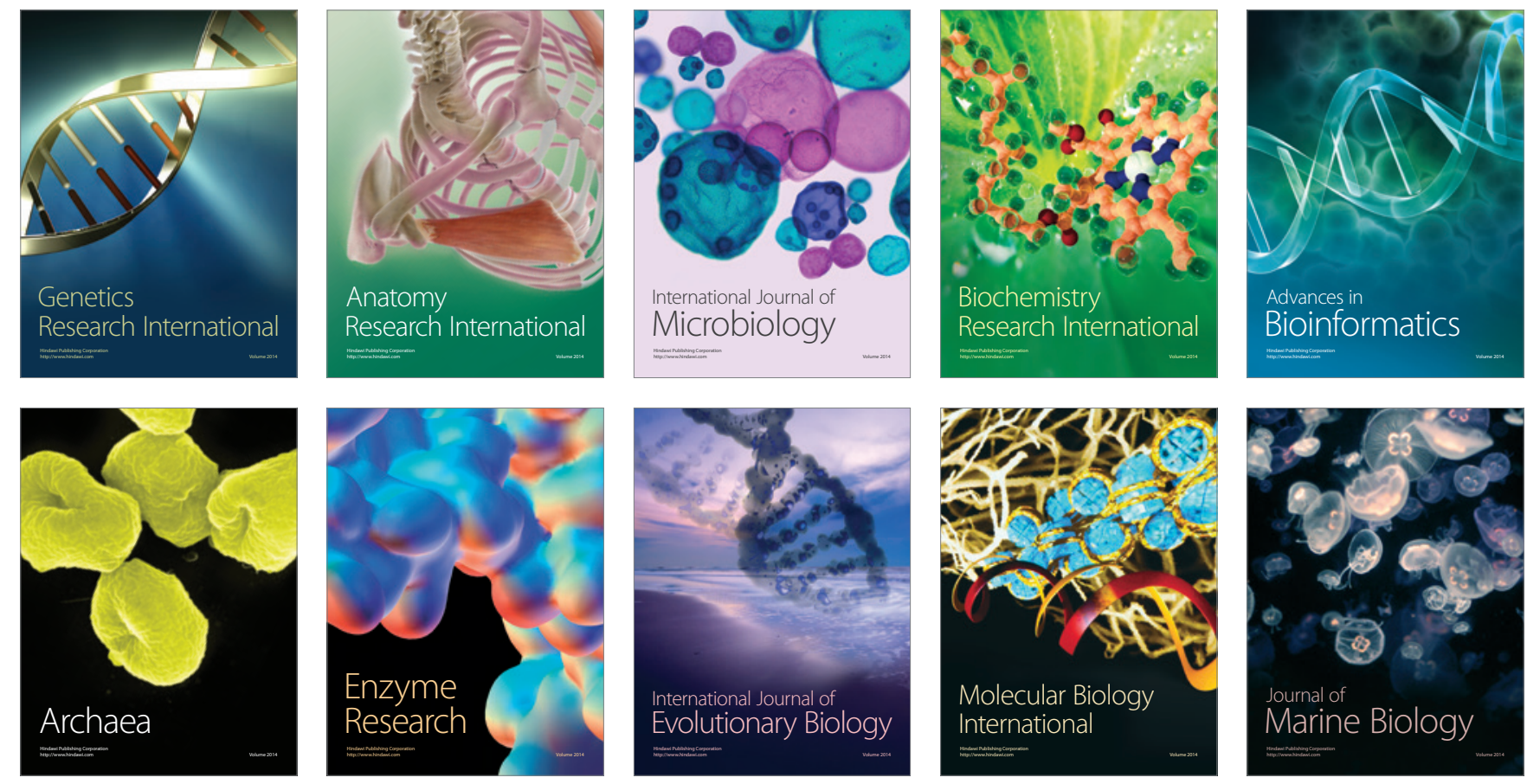